\title{
Parametric $x$-ray radiation and coherent bremsstrahlung from nonrelativistic electrons in crystals
}

\author{
I. D. Feranchuk, ${ }^{1}$ A. Ulyanenkov, ${ }^{2}, *$ J. Harada, ${ }^{2}$ and J. C. H. Spence ${ }^{3}$ \\ ${ }^{1}$ Byelorussian State University, F. Skariny Avenue, 4, 220050 Minsk, Republic of Belarus \\ ${ }^{2} X$-ray Research Laboratory, Rigaku Corporation, 3-9-12 Matsubara-cho, Akishima-shi, Tokyo 196-8666, Japan \\ ${ }^{3}$ Department of Physics, Arizona State University, Tempe, Arizona 85287 \\ (Received 28 October 1999; revised manuscript received 24 April 2000)
}

\begin{abstract}
A theoretical analysis of radiation spectra produced during the coherent interaction of nonrelativistic electrons with crystals has been carried out. The output intensity has been found to be the result of interference between two distinguishable phenomena, coherent Bremsstrahlung and parametric x-ray radiation. The latter is determined by a coherent summation of transition radiation from electrons interacting with successive crystallographic planes. The interference is shown to be considerable for the case of nonrelativistic electrons, and so allows us to describe quantitatively the experiments of Korobochko et al. (Zh. Éksp. Teor. Fiz. 48, 1248 (1965) [Sov. Phys. JETP 21, 834 (1965)]) and Reese et al. [Philos. Mag. A 49, 697 (1984)]. The conditions for possible application of coherent $\mathrm{x}$-ray radiation, a comparison with synchrotron radiation, and the requirements for experimental setup are discussed.

PACS number(s): 41.60.Bq, 98.70.Qy, 41.50.+h
\end{abstract}

\section{INTRODUCTION}

There are several recognized mechanisms of x-ray production by electrons moving inside a crystal. One of them, called parametric x-ray radiation (PXR), was considered for the first time by Ter-Mikaelian [1] in the framework of perturbation theory, which is valid for particles passing through a thin crystal. The radiation described in [1] can be interpreted as coherent transition radiation from electrons uniformly moving within the crystal. Baryshevskii and Feranchuk [2] and Garybyan and Yang [3] have further shown that a more adequate description of this phenomenon is based on the diffraction of the electron's electromagnetic field in a crystal. The theory $[2,3]$ comprehensively explains PXR production from electrons within crystals of arbitrary thickness. The experimental observations of PXR have been reported both for ultrarelativistic electrons with energy of hundreds $\mathrm{MeV}$ [4-6] and for relatively low-energy but still relativistic electrons of 5-10 MeV [7]. The results of these experiments confirm the theoretical prediction of PXR intensity attenuation with respect to decrease of electron energy. However, the intensity of PXR still remains high [8] when using nonrelativistic particles with energy of hundreds $\mathrm{keV}$. Such an $\mathrm{x}$-ray beam may serve as a quasimonochromatic $\mathrm{x}$-ray source with tunable frequency and can be utilized for laboratory x-ray studies, which are presently conducted only with synchrotron radiation.

Coherent Bremsstrahlung (CBS), the other widely recognized orientational effect yielding $\mathrm{x}$-rays, is related to the coherent scattering of electrons on the periodic atomic structure of crystal. This radiation has been comprehensively studied both theoretically (see [1] and citations therein) and

\footnotetext{
*On leave from the Institute of Nuclear Problems, Minsk, Republic of Belarus. Corresponding author. Email address: Alex@Rigaku.co.jp
}

experimentally $[1,9,10]$. In most experiments, however, relativistic and ultrarelativistic electrons were used that lead to the CBS characteristic frequencies appearance in the region of hard $\gamma$ radiation with energy of $\mathrm{MeV}$. There are only experiments by Korobochko [11] et al. and Reese [12] et al., that used nonrelativistic electrons. To our knowledge, a detailed interpretation of the above-mentioned experiments, which could clarify the mechanism of observed x-ray radiation, has not been given previously. The aim of the present work is the theoretical analysis of $\mathrm{x}$-ray spectra from nonrelativistic electrons coherently interacting with the crystal. A peculiarity of the energy interval considered for the electrons is the specific angular distribution of radiation, which requires improvement and optimization of the detection scheme used for relativistic particles.

In the case of relativistic or ultrarelativistic particles passing through a crystal, the background radiation caused by effects other than PXR, for example, coherent and incoherent Bremsstrahlung, is concentrated within a narrow cone along the direction of the particle beam (Fig. 1a). Quasimonochromatic photon beams appearing in directions determined by Bragg angles with respect to the crystallographic planes arise due to the PXR phenomenon only [13]. As a result, even detectors with relatively low spectral resolution are able to record easily the PXR maxima in the vicinity of Bragg directions. In the case of nonrelativistic electrons, the angular distribution of radiation caused by all generation mechanisms, including PXR and CBS, is almost isotropic (Fig. 1b). Therefore, peaks originating from coherent orientational effects are observed on an intense uniform background, and their shapes and amplitude are mainly defined by the spectral resolution of detector. Thus, the relativistic factor plays a principal role in the angular distribution of emitted photons.

One more physical effect gaining strength in the nonrelativistic case is the interference of PXR and CBS amplitudes, recently discussed in papers [14-16]. The general possibility for amplitude interference of different radiation modes follows from their uniform description by the same matrix ele- 


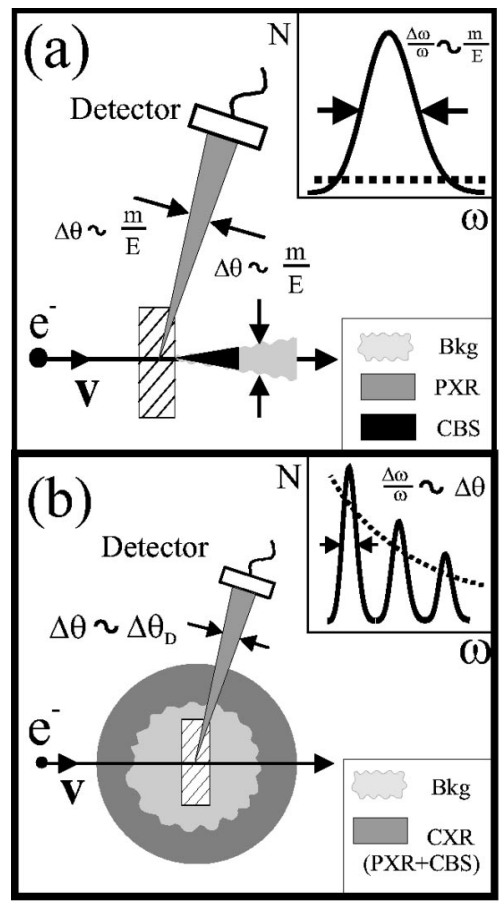

FIG. 1. The spatial distributions of $\mathrm{x}$-ray radiation intensity for relativistic (a) and nonrelativistic (b) electrons. In the relativistic case, the background (Bkg) and coherent Bremsstrahlung (CBS) are concentrated in narrow $(\theta \approx m / E)$ cone whose axis coincides with the electron velocity vector. Parametric $\mathrm{x}$-ray radiation (PXR) can be easily detected in the directions determined by the Bragg condition for crystallographic planes of the sample. In the nonrelativistic case, the background and coherent $\mathrm{x}$-ray radiation, consisting of interfering PXR and CBS are distributed almost isotropically. The insets show the spectral structure of the detected peaks.

ments of the S matrix using an appproach of quantum electrodynamics, as was pointed out by Baryshevskii and Feranchuk [17]. In particular, the interference of PXR and channeling radiation has been considered by Baryshevskii [18], in the framework of such an approach. However, the radiation kinematics of relativistic particles assumes the supression of interference between PXR and other effects. For instance, the CBS amplitude is considerably smaller than the PXR amplitude (their ratio is determined by the relativistic factor $[14,15])$. Nevertheless, the effect was recently shown [16] to be observable for electrons with energy of a few $\mathrm{MeV}$.

In the present work, we demonstrate that interference of PXR and CBS reaches its maximum for nonrelativistic electrons, merging these processes on the basis of uniform amplitude of coherent $\mathrm{x}$-ray radiation $(\mathrm{CXR})$. Taking into account this fact along with the abovementioned detector resolution effect is found to be necessary for a quantitative description of experiments $[11,12]$. The paper is structured as follows. In Sec. II we derive a general expression for the coherent radiation spectrum produced by nonrelativistic electrons passing through a crystal and give some numerical examples of ideal radiation spectra. In Sec. III we take into account real experimental conditions, the spatial and spectral resolution functions of detector. The spectra simulated on the basis of the derived formulas are then compared with experimental data $[11,12]$. Section IV compares the intensities of
CXR and synchrotron radiation. The formulas derived permit one to estimate the possibility of using coherent $\mathrm{X}$-ray radiation as a potential source of $\mathrm{x}$-rays. Conclusions are presented in Sec. V.

\section{COHERENT RADIATION FROM NONRELATIVISTIC ELECTRONS IN CRYSTAL}

A rigorous expression for the cross section of electrodynamic processes within the crystal has been derived [17] from quantum electrodynamics (QED) and takes into account the coherent interaction of both electrons and photons with the media (see also earlier work [19] by the same authors). However, it makes sense to use a general equation only if (i) recoil effect must be taken into account in radiation of hard photons, and (ii) the quantization of electrons' transverse motion is considered (channeling radiation). Both reasons are important in the case of relativistic electrons, but if these effects are negligible (nonrelativistic case), the quasiclassical limit of general QED expression can be used. Then we obtain the following expression for the spectral density of the photon number emitted in direction $\mathbf{n}$ (for details of this derivation, the reader is refered to the monograph of Baryshevskii [18]).

$$
d^{2} N_{\mathbf{n} \omega}^{(s)}=\frac{e^{2} \omega}{4 \pi^{2}}\left|\int_{0}^{t_{L}} d t \quad \mathbf{v}(t) \cdot \mathbf{E}_{\mathbf{k} s}[\mathbf{r}(t), \omega] e^{-i \omega t}\right|^{2} d \omega d \mathbf{n}
$$

The Eq. (1) takes into account the interaction of both the electron and the electromagnetic field of the emitted photons with the crystal. Here $\omega$ and $\mathbf{k}=\omega \mathbf{n}$ are the frequency and wavevector of radiation in the direction $\mathbf{n}$, respectively. The units are chosen so $\hbar=c=1 ; \mathbf{r}(t)$ and $\mathbf{v}(t)$ are the coordinates and the velocity of the electron within the crystal, respectively; $\mathbf{E}_{\mathbf{k} s}[\mathbf{r}(t), \omega]$ is the wavefield of the emitted electromagnetic wave with well-defined polarization, which should be found by taking into consideration the interaction of the wavefield with the crystal. The intervals $d \omega$ and $d \mathbf{n}$ define the spectral and angular area where the photons are detected, and $t_{L}$ represents the time over which the electron moves inside the crystal of thickness $L$. In the experiments of Refs. [11,12], thin crystalline films were used, enabling one to find the functions $\mathbf{r}(t)$ and $\mathbf{E}_{\mathbf{k} s}(\mathbf{r}, \omega)$ by perturbation theory. This is important for a physical interpretation of the results because: i)the contribution by different x-ray generation modes to the radiation amplitude can be considered additively, ii) multiple scattering of electrons in the crystal may be neglected when considering the formation of the coherent radiation peaks (for calculations of the CBS emission spectra from diamond that include multiple scattering of the electron beam and the contributions of individual lattice planes, and the effects of these emission lines on energy-loss spectra, see Spence and Reese [20]).

The expression for the electromagnetic field of the emitted radiation under specific diffraction conditions is well known [8,21]: 


$$
\begin{aligned}
& \mathbf{E}_{\mathbf{k} s}(\mathbf{r}, \omega)=\mathbf{e}_{s} \exp (i \mathbf{k} \cdot \mathbf{r})+\sum_{\mathbf{g} \neq 0} \mathbf{E}_{\mathbf{g} s} \exp [i(\mathbf{k}+\mathbf{g}) \cdot \mathbf{r}], \\
& \mathbf{E}_{\mathbf{g} s}=-\frac{\chi_{g}}{\left[k_{g}^{2}-\omega^{2}\right]}\left[\mathbf{k}_{g} \cdot\left(\mathbf{g} \cdot \mathbf{e}_{s}\right)-\omega^{2} \mathbf{e}_{s}\right], \quad \mathbf{k}_{g}=\mathbf{k}+\mathbf{g},
\end{aligned}
$$

where $\mathbf{e}_{s}$ is the polarization vector; $\chi_{g}$ are the Fourier components of the polarizability describing the coherent interaction of the emitted radiation with periodically distributed electron density of the crystal. In fact, this interaction defines the main characteristics of PXR. The summation is performed over all reciprocal lattice vectors $\mathbf{g}$ and the volume of the sample is assumed to be unity.

Assuming the radiation frequency to be far from the characteristic frequencies of the crystal atoms, the Fourier components of polarizability can be calculated according to

$$
\begin{array}{r}
\chi_{g}=-\frac{4 \pi e^{2}}{m \omega^{2}} \frac{S(\mathbf{g})}{\Omega} \exp [-W(\mathbf{g})] \\
S(\mathbf{g})=\sum_{i} F_{i}(\mathbf{g}) \exp \left(i \mathbf{g} \cdot \mathbf{R}_{i}\right) .
\end{array}
$$

Here $e$ and $m$ are the charge and the mass of the electron, respectively; $S(\mathbf{g})$ is the structure factor of the crystal elementary cell of volume $\Omega$, evaluated as a sum of form factors $F_{i}(\mathbf{g})$ of separate $i$ th atoms at the positions $\mathbf{R}_{i} ; \exp [-W(\mathbf{g})]$ is the Debye-Waller factor taking into account thermal vibrations of atoms. It should be noted, that formula (1) for the intensity of radiation from low-energy electrons is applicable for a crystal of arbitrary thickness because the kinematics of PXR assumes the vector $\mathbf{k}$ is always far from the Ewald sphere and dynamical effects are negligible $[8,21]$.

Evidently, the interaction of electrons and electromagnetic radiation with a crystal results not only in changing the stationary states of the electromagnetic field but in varying the motion law $\mathbf{r}(t)$ of the electron as well. The generation of CBS is caused by the scattering of electrons by a coherent periodic potential, which is defined by the Coulomb interaction of the beam particles both with the electron density of the crystal and with the nuclei. This potential can be written as [1]:

$$
\begin{gathered}
U(\mathbf{r})=\frac{1}{\Omega} \sum_{\mathbf{g} \neq 0} U_{\mathbf{g}} \exp (i \mathbf{g} \cdot \mathbf{r}), \\
U_{\mathbf{g}}=4 \pi e \sum_{i} \exp \left(i \mathbf{g} \cdot \mathbf{R}_{i}\right) \frac{\left[Z_{i}-F_{i}(\mathbf{g})\right]}{g^{2}} \exp [-W(\mathbf{g})],
\end{gathered}
$$

where $Z_{i}$ is the charge of the atomic nucleus in the $i$ th position of crystal elementary cell, and the other notations have the same meaning as in formula (3). The law of motion $\mathbf{r}(t)$ of an electron in a potential (4) can be found solving Newton equations with an accuracy justified up to $O\left(U_{\mathbf{g}}\right)$ :

$$
\mathbf{r}(t)=\mathbf{r}_{0}+\mathbf{v}_{0} t+\mathbf{r}_{1}(t),
$$

$$
\mathbf{r}_{1}(t)=i \frac{e}{m} \sum_{\mathbf{g}} \frac{\mathbf{g}}{\left(\mathbf{g} \cdot \mathbf{v}_{0}\right)^{2}} U_{\mathbf{g}} \exp \left(i \mathbf{g} \cdot \mathbf{v}_{0} t\right)
$$

The velocity of the electron beam in vacuum is designated here as $\mathbf{v}_{0}$. The inequality $r_{1} \ll v_{0} t$ defines the validity region of the subsequent calculations of radiation intensity. Substituting Eq. (5) into this inequality, the expression for the range of validity can be found to be

$$
\frac{4 \pi e^{2} Z}{m g^{2} \Omega} \omega L \ll 1 .
$$

Here $Z$ is the averaged electrical charge per single atom. In fact, the condition (6) means that a restriction to the length of a particle trajectory within the crystal has the same value as the extinction length of emitted photons, and we can apply the current classical approach to relatively thick crystals. From the quantum point of view, this can be explained as follows. Certainly, the wavefunction of a particle, being a part of the matrix element for radiation intensity, is determined by the essentially less extinction length for the electrons. But for a crystal of thickness not exceeding the photon extinction length, the considerable phase oscillations of the electron wave functions are mutually canceled in the initial and final states of the particle. Thus, within the limits of the classical approach, the calculation of radiation intensity is free of difficulties related to the necessary calculation of multiwave diffraction of electrons when the quantum theory is used [12]. A comparison of the energy flow in the classical and quantum pictures has been given in Ref. [22].

After the substitution of the expression in Eq. (5) into Eq. (1), an expression for the radiation intensity in a thin crystal can be derived with an accuracy up to $O\left(U_{\mathbf{g}}\right)$

$$
\frac{\partial^{2} N_{\mathbf{n}, \omega}^{(s)}}{\partial \omega \partial \mathbf{n}}=\frac{e^{2}}{4 \pi^{2}} \omega \sum_{\mathbf{g} \neq 0}\left|A_{\mathbf{g s}}(\omega, \mathbf{n})\right|^{2},
$$

where the amplitudes $A_{\mathrm{gs}}$ are defined by the following formula (the $z$ axis is chosen to be parallel to the velocity vector $\left.\mathbf{v}_{0}\right)$ :

$$
\begin{gathered}
A_{\mathbf{g s}}=\left\{\mathbf{v}_{0} \cdot \mathbf{E}_{\mathbf{g} s}-\frac{e}{m} \frac{U_{\mathbf{g}}}{\mathbf{g} \cdot \mathbf{v}_{0}}\left[\mathbf{e}_{s} \cdot \mathbf{g}+\left(\mathbf{e}_{s} \cdot \mathbf{v}_{0}\right) \frac{\mathbf{k} \cdot \mathbf{g}}{\mathbf{g}_{s} \cdot \mathbf{v}_{0}}\right]\right\} Q, \\
Q=\frac{\sin q L_{z} / v_{0}}{q}, \quad q=\frac{\omega-\mathbf{v}_{0} \cdot(\mathbf{k}+\mathbf{g})}{2} .
\end{gathered}
$$

The first term in formula (8) describes PXR, whereas the second term determines the coherent Bremsstrahlung. The position of the intensity peaks in Eq. (8) is defined by the same kinematic factor $|Q|^{2}$, which appears due to coherent interference of radiation formed by different crystallographic planes. A similar factor was considered for the kinematical model of PXR from relativistic electrons [13], and the contribution of this factor to intensity can be represented as

$$
|Q|^{2}=2 \pi \frac{L_{a}}{v_{0}}\left[1-e^{-L_{z} / L_{a}}\right] \delta\left[\omega-\mathbf{v}_{0} \cdot(\mathbf{k}+\mathbf{g})\right]
$$


Here $L_{a}$ is the absorption length of the crystal for the frequency determined from the zeros of $\delta$-function argument in Eq. (9). Thus, the spectral and angular distributions of radiation, which result from coherent processes initiated by a nonrelativistic electron beam inside a thin crystal are defined by the sum of resonant terms. The most important point is that these terms impose the same conditions on the frequency and the direction of the emitted photons both for parametric x-ray radiation and coherent Bremsstrahlung. For every selected crystallographic reflection with interplane distance $d$, a set of narrow spectral lines with frequencies $\omega_{n}(\theta)$ is formed in the selected direction with observation angle $\theta$ to the velocity vector of electron [8]:

$$
\omega_{n}(\theta)=\frac{2 \pi v_{0} \cos \theta_{B}}{d\left(1-v_{0} \cos \theta\right)} n, \quad n=1,2, \ldots
$$

The relative width of these lines is

$$
\frac{\Delta \omega_{0}}{\omega} \approx \frac{v_{0}}{L_{z} \omega_{n}(\theta)}
$$

where $\theta_{B}$ is the angle between the velocity $\mathbf{v}_{0}$ and the normal to the crystallographic planes taking part in the scattering process. For nonrelativistic electrons, the number of emitted photons depends weakly on the variation of angle $\theta$ and is defined by the sum of the interfering amplitudes of PXR and CBS. The number of photons emitted in the chosen direction integrated in the vicinity of the peak and within frequency interval $\Delta \omega>\Delta \omega_{0}$ is expressed for $L_{z} \leqslant L_{a}$ as

$$
\frac{\partial N_{s}}{\partial \mathbf{n}} \simeq \frac{e^{2}}{2 \pi} \omega_{n} \frac{L_{z}}{v_{0}}\left|A_{P X R}+A_{C B S}\right|^{2}
$$

with

$$
\begin{gathered}
A_{P X R}=\frac{\chi_{g}}{\left[k_{g}^{2}-\omega^{2}\right]}\left[\left(\mathbf{v}_{0} \cdot \mathbf{k}_{g}\right)\left(\mathbf{g} \cdot \mathbf{e}_{s}\right)-\omega^{2}\left(\mathbf{v}_{0} \cdot \mathbf{e}_{s}\right)\right], \\
A_{C B S}=-\frac{e U_{g}}{m \Omega\left(\mathbf{g} \cdot \mathbf{v}_{0}\right)}\left[\mathbf{g} \cdot \mathbf{e}_{s}+\left(\mathbf{v}_{0} \cdot \mathbf{e}_{s}\right) \frac{\mathbf{k} \cdot \mathbf{g}}{\mathbf{v}_{0} \mathbf{g}}\right] .
\end{gathered}
$$

The general formulas (12)-(14) have the same meaning as the resulting expressions obtained recently in the paper by Morokhovskyi et al., [16] which also deals with the radiation of electrons, coherently interacting with a crystal. However, our derivation seems to be more useful for clarification of the mechanism and origin of the radiation, and emphasizes the meaning of the approximations used. In comparison with the case of relativistic particles [16], a new physical result follows from the application of formulas (12)-(14) to the analysis of radiation from nonrelativistic particles, consisting in a considerably different ratio of PXR and CBS amplitudes. For relativistic particles, this ratio depends on the electron energy (in experiment Ref. [16] the amplitude of CBS is one half of PXR amplitude for electrons of $4 \mathrm{MeV}$ ), whereas for nonrelativistic particles the ratio of the first to the second term in Eq. (12) depends only on the distribution of charge density within the elementary crystal cell (see discussion below). Moreover, obviously marked peaks, used in the experiment of Ref. [16] for analysis of PXR/CBS interference, are absent in the angular distribution of radiation from nonrelativistic electrons. This makes it necessary to take precisely into account the instrumental function of the detector and incoherent Bremsstrahlung background, when performing a theoretical analysis of experimental curves (Sec. III).

The Eqs. (13) and (14) explain the role of the relativistic factor in the formation of coherent peaks. The expression in the denominator of formula (13)

$$
\delta=k_{g}^{2}-\omega_{n}^{2}=2 \omega_{n}(\mathbf{n} \cdot \mathbf{g})+g^{2} \simeq g^{2}\left(1-\frac{v_{0} \cos \theta_{B}}{1-v_{0} \cos \theta}\right),
$$

describes the deviation of the emitted photon wavevector from the Ewald sphere. For the nonrelativistic case, when the condition $1-v_{0} \approx 1$ is fulfilled, the value of the parameter $\delta$ is approximated by $\delta \approx g^{2}$, and the contributions of PXR and CBS to coherent peaks are of the same order. As the energy of the electron $E$ increases with $v_{0} \rightarrow 1$, the parameter $\delta$ becomes negligible for some angles $\theta$ and the intensity of PXR increases [8] proportionally to $(E / m)^{2}$, reaching its maximum at $E_{\text {opt }} \sim m / \sqrt{\chi_{g}}$ (see [2] for details).

In the present work, interest is focused on PXR from nonrelativistic electrons and possible applications of this radiation [8]. The angular distribution of x-ray radiation caused by a given set of crystallographic planes in this case is almost isotropic and we may neglect the slight difference in dependence of the polarization terms in Eqs. (13) and (14) on angle $\theta$. Using Eq. (10) for the resonant frequency, the ratio of PXR and CBS contributions to the coherent peak is obtained as follows:

$$
\delta_{g}=\frac{A_{P X R}}{A_{C B S}} \simeq \frac{\sum_{i} F_{i}(\mathbf{g}) \exp \left(i \mathbf{g} \cdot \mathbf{R}_{i}\right)}{\sum_{i}\left[Z_{i}-F_{i}(\mathbf{g})\right] \exp \left(i \mathbf{g} \cdot \mathbf{R}_{i}\right)} .
$$

The Eq. (16) reflects the physical nature of peak formation in the radiation spectra. Parametric x-ray radiation contributes to peaks due to the coherent scattering of emitted photons by the atomic electrons only, whereas the CBS is caused by the coherent scattering of incident charged particles both by electrons and nuclei.

The formulas (12)-(14) derived in this section define the position and relative amplitudes of lines in the spectral series, which can be considered as ideal spectra of coherent $\mathrm{X}$-ray radiation from nonrelativistic electrons in a crystal. Figure 2 shows the spectra simulated on the basis of the presented theory for crystals of $\mathrm{Si}(\mathrm{a}, \mathrm{b}), \mathrm{MgO}(\mathrm{c}, \mathrm{d})$ and $\mathrm{LiF}$ $(e, f)$. The panels (a) and (c) correspond to the electron velocity parallel to the $\langle 111\rangle$ crystallographic axis, (b) and (d); parallel to $\langle 100\rangle$, curves (e) and (f) are calculated for electrons striking the crystal perpendicular to the (110) and (100) planes, respectively. Only the reflections contributing essentially to peak intensities are depicted. The symbols (hkl) denote single crystallographic plane, and $\{\mathrm{hkl}\}$ means a crystal form (set of planes). The ideal spectra illustrated in Fig. 2 demonstrate the positions of CXR peaks, their absolute intensities and the contribution of PXR intensity (black part of bar) to the full CXR output (full bar). The left panels are drawn on a logarithmic scale to emphasize the intensity re- 


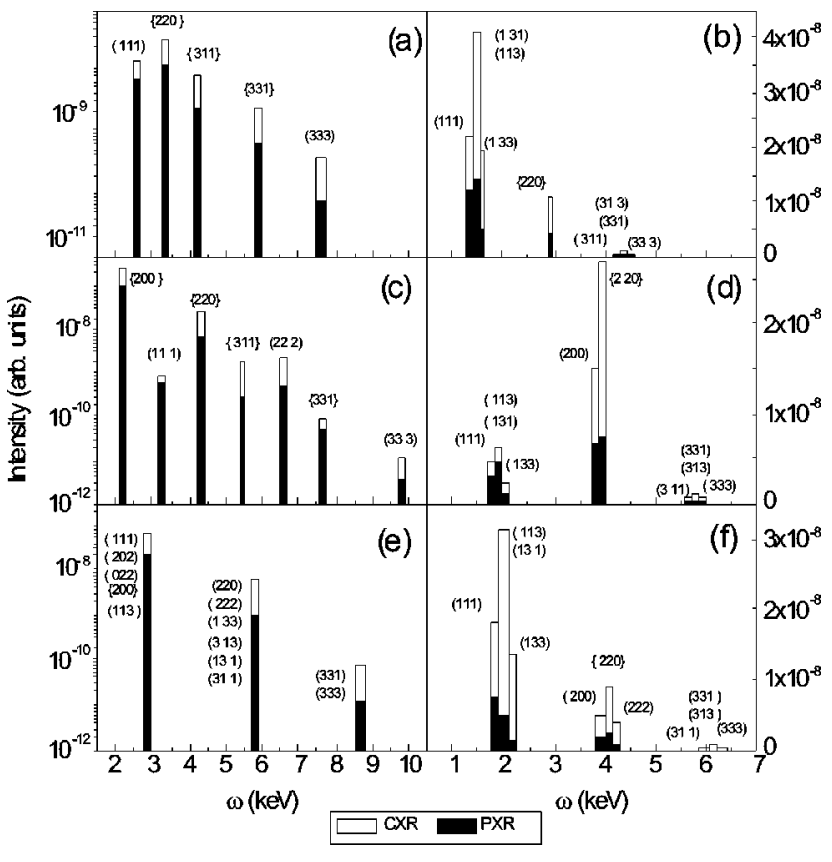

FIG. 2. The most intense series of CXR peaks for $\mathrm{Si}(\mathrm{a}, \mathrm{b}), \mathrm{MgO}$ $(\mathrm{c}, \mathrm{d})$ and $\operatorname{LiF}(\mathrm{e}, \mathrm{f})$ crystals taken at different orientations of samples with respect to the electron velocity. The diagrams illustrate the positions of peaks and contribution of PXR (filled area) to the integral CXR intensity. The parameters for the electron beam and crystal orientation are chosen to be similar to experimental conditions of Refs. $[11,12]$. In the right panels the peaks are split in intensities from different reflections. The corresponding individual reflections and sets of crystal planes contributed to peaks are depicted near bars. For details see the text.

cession in the high-order peak series. In the right panels, the contributions of radiation produced by different crystallographic planes to CXR lines are separated. Moreover, the intensity scale is chosen to be linear for the representation of the real PXR/CXR ratio in the output signal. The crystals and experimental conditions used for simulations are assumed to be the same as in Refs. [11,12]. The diagrams for $\mathrm{Si}$ and $\mathrm{MgO}$ are calculated for electrons of energy $\mathrm{E}=120 \mathrm{keV}$ and an observation angle $\theta_{0}=96^{\circ}$, the spectra for $\mathrm{LiF}$ single crystal are simulated for $\mathrm{E}=84 \mathrm{keV}$ and $\theta_{0}=67.5^{\circ}$. The fine spectral and angular structure of peaks will be studied in detail in the next section.

\section{SIMULATION OF REAL RADIATION SPECTRA}

The principal difference between the above derived ideal CXR spectrum resulting from the interference of PXR and the CBS from nonrelativistic particles and PXR spectrum from ultrarelativistic particles [13] is that the low-energy particles emit the radiation isotropically, whereas the angular distribution of the ultrarelativistic PXR represents the set of reflections with divergence determined by relativistic factor $m / E$ (Fig. 1a). Another important point is that in the relativistic case the incoherent interaction of electrons with the crystal causes the radiation to be concentrated within a narrow cone prolated along the particle motion direction. Therefore, the intensity of PXR exceeds considerably the incoherent background when the X-rays are observed in directions different from the velocity of electrons. Under this condition, the PXR peaks can be recorded even with detectors of relatively low spectral resolution. Coherent Bremsstrahlung from relativistic particles spreads in the vicinity of electronbeam direction and its spectrum shifts to hard x-ray region. In the experiments with nonrelativistic electrons (Fig. 1b), the CXR peaks, being composed of PXR and CBS contributions, are accompanied by an intense background caused by other radiation types, e.g., the incoherent Bremsstrahlung. The angular distribution of the latter is almost isotropic for nonrelativistic particles. Therefore, the investigation of the spectral distribution of CXR peaks at the fixed angle of observation requires the detectors with high energy resolution, or additional analyzers that are usually used to monochromatize synchrotron radiation [24].

To define the requirements for the detector, the spectral intensities of coherent X-ray radiation and Bremsstrahlung (BS) must be estimated. Comparing the intensities, we disregard the kinematical factors related to the weak dependence of intensity on radiation angle, i.e., the angle distributions of both CXR and BS are assumed to be isotropic. This allows one to emphasize the most essential dimensionless parameters affecting the ratio of CXR and BS output. Using the formula (28.4.2) for BS spectrum from [25], the estimation for Bremsstrahlung can be written as

$$
\frac{\partial N_{s}}{\partial \mathbf{n}} \simeq \frac{4 e^{2}}{3 \pi} Z^{2}\left(\frac{e^{2}}{m}\right)^{2} \rho L_{z} \ln \left[\frac{137}{Z^{1 / 3}}\right] \frac{\Delta \omega}{\omega},
$$

where $\rho$ is the concentration of scattering centers $(\rho=1 / \Omega$ for crystals with a single atom per elementary cell). We consider here only photons originating from Bremsstrahlung and emitted near one of the CXR peaks. To estimate roughly the ratio of quanta number in a CXR peak to quanta number of incoherent BS, the amplitudes of PXR and CBS in Eq. (12) are assumed to be equal and do not depend on angles. Then, using the explicit expression (3) for polarizability, the estimation is

$$
\frac{\partial N_{s}}{\partial \mathbf{n}} \simeq \frac{e^{2}}{2 \pi} \omega_{n} L_{z} v_{0}\left[\frac{4 \pi e^{2}}{m \omega^{2}} \frac{S(\mathbf{g})}{\Omega} \exp [-W(\mathbf{g})]\right]^{2}
$$

If the crystals with a single atom per unit cell are considered,

$$
\frac{S(\mathbf{g})}{\Omega} \exp [-W(\mathbf{g})] \simeq \rho Z,
$$

and the ratio of CXR to BS intensities within the limits of approximations used above can be found:

$$
\eta=\frac{\left[\partial N_{s} / \partial \mathbf{n}\right]_{C X R}}{\left[\partial N_{s} / \partial \mathbf{n}\right]_{B S}} \simeq \frac{\rho}{\omega_{n}^{3}} \frac{6 \pi^{2} v_{0}}{\ln \left[137 / Z^{1 / 3}\right]} \frac{\omega_{n}}{\Delta \omega} .
$$

Thus, the ratio of intensities in the vicinity of a peak is determined by the coherency factor

$$
\xi_{n}=\frac{\rho}{\omega_{n}^{3}},
$$

that results from the interference of radiation generated on the periodic atomic structure. For example, for experiment 
[11] with an $\mathrm{LiF}$ crystal and electrons of energy $63 \mathrm{KeV}$ penetrating the crystal parallel to the [100] crystallographic axis and observation angle $\theta=67.5^{\circ}$ the coherency factor $\xi$ $\simeq 0.39$ for spectral series related to (200) reflection, and $\xi$ $\simeq 0.79$ for (111) reflection. To distinguish spectral peaks from uniform background, the detector has to have a resolution satisfying the condition $\xi_{1} \gg 1$ :

$$
\xi_{1}=\xi \frac{\omega}{\Delta \omega},
$$

parameter $\xi_{1}$, describing the ratio signal/background in the spectrum reaches its maximum value when the resolution of the detector is the same order as the linewidth of CXR, as formula (11) states. In the real experiments, such a resolution can be achieved by using a crystal analyzer. Assuming the same conditions as in experiment [11], the maximum value of parameter $\eta$ for reflection (200) is approximately equal to

$$
\eta_{\max } \simeq \frac{\rho}{\omega_{n}^{3}} \frac{6 \pi v}{\ln \left[137 / Z^{1 / 3}\right]} \omega_{n} L_{z} \simeq 1.3 \times 10^{3},
$$

if the spectral resolution of the detector is assumed to be about $0.1 \%$. The resolution of real detectors in the experiments $[11,12]$ was only about $10 \%$. The better is the spectral resolution of detector, the larger is the coherency factor, in according with formula (18), and the higher is the relative magnitude of CXR peaks in comparison to background of incoherent Bremsstrahlung. This result has been recently confirmed in experiments [23], where the low-energy part of radiation spectrum was recorded using a crystal spectrometer with $40 \mathrm{eV}$ resolution and a new fine structure of peaks has been revealed.

The absolute number of photons emitted by one electron and contributing to the CXR peak is essentially smaller than the PXR intensity from ultrarelativistic electrons [see discussion in Ref. [8]]. However, due to the considerably higher current density which can be achieved for nonrelativistic particles in comparison to linear accelerator beams, the integrated number of quanta is easily detectable in the former case. Assuming, again, the parameters of experiment [11], i.e. the electron current and energy to be equal $1 \mu \mathrm{A}$ and 63 $\mathrm{KeV}$, respectively, a $100-\mathrm{nm}$ thick LiF crystal, velocity of electrons to be parallel to [100] axis, the estimation for absolute number of detected photons in the vicinity of the fundamental peak from (200) reflection is given by Eq. (12):

$$
N_{0} \simeq 5.1 \times 10^{2} \text { photons } / \mathrm{sec}, \quad \omega_{0} \simeq 3.89 \mathrm{keV} .
$$

Here the values for the Bragg angle, $\theta_{B}$, and the observation angle, $\theta$, are taken as $\theta_{B}=0 ; \theta=67.5^{\circ}$, and the detector registers the photons in the solid angle defined by deviation of the normal vector in the region of $\Delta \mathbf{n} \sim 10^{-3}$ steradian. This estimation (22) is in good agreement with radiation intensity observed in [11].

Let us turn now to calculation of real CXR spectra taking into account the convolution of ideal spectra with both the angular and spectral resolution functions of the detector and the background of incoherent radiation. To evaluate the mentioned integration, the following substitution is used, in accordance with Fig. 3:

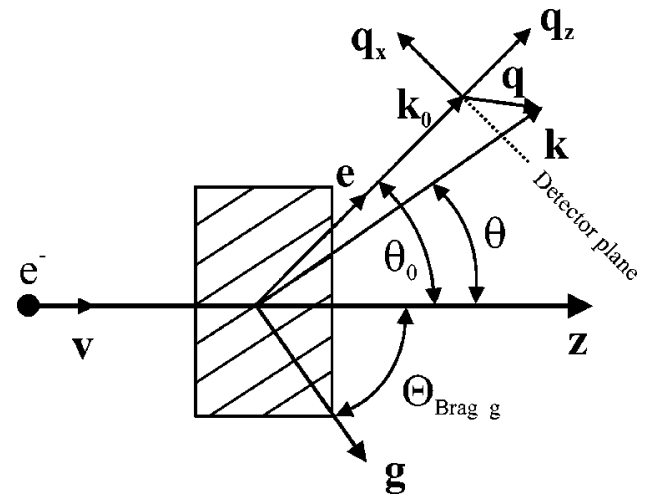

FIG. 3. Geometrical sketch of vectors and angles describing the PXR and CBS processes.

$$
\mathbf{k}=\mathbf{k}_{0}+\mathbf{q} ; \quad \mathbf{k}_{0}=\omega_{g} \mathbf{e}
$$

Here vector e defines the direction of the detector center and the parameter

$$
\omega_{g}=\frac{v \cos \theta_{B}}{\left(1-v \cos \theta_{0}\right)} g
$$

determines the frequency of a peak in ideal CXR spectra, corresponding to reciprocal lattice vector $\mathbf{g}$. The instrumental functions describing the angular and spectral resolutions of the detector are

$$
\begin{gathered}
f_{1}(\theta)=\frac{1}{\pi} \exp \left[-\frac{\left(\theta-\theta_{0}\right)^{2}}{\Delta \theta^{2}}\right], \\
f_{2}(\omega)=\frac{1}{\sqrt{\pi}} \exp \left[-\frac{\left(\omega-\omega_{r}\right)^{2}}{\Delta \omega^{2}}\right],
\end{gathered}
$$

where $\Delta \theta$ is the angle aperture of detector which is assumed, for simplicity, to be a pin-hole slit, $\Delta \omega$ is the detector spectral resolution, the frequencies $\omega_{r}=r \varepsilon(r=0,1,2 \ldots)$ correspond to $r$-th detector channel with width $\varepsilon$. Because in real experiments $\varepsilon<\Delta \omega \ll \omega_{r}$ and $\Delta \theta \ll \theta_{0}$, the phase volume of the detected photons can be expressed via new variables as

$$
\begin{gathered}
\omega \simeq \omega_{g}+q_{z}+\frac{q^{2}}{2 \omega_{0}}, \\
d \mathbf{k}=d \mathbf{q} \simeq \omega_{r}^{2} \eta d \eta d \varphi d \nu, \\
\nu=\omega-\omega_{r}, \quad \eta=\theta-\theta_{0},
\end{gathered}
$$

where the $z$ axis coincides with vector $\mathbf{k}_{0}$ and $\varphi$ is the angle between vector $q_{\perp}$ and the axis perpendicular to the plane defined by vectors $\mathbf{v}_{0}$ and $\mathbf{g}$. Then the real spectrum of CXR approximating the conditions of experiments $[11,12]$ may be written as follows: 


$$
\begin{aligned}
N_{r}= & B \Delta \theta^{2} \frac{\Delta \omega}{\omega_{r}}+\frac{1}{\pi^{3 / 2}} \int_{0}^{\infty} \eta d \eta \int_{0}^{2 \pi} d \varphi \\
& \times \int_{-\infty}^{\infty} d \nu \exp \left[-\frac{\eta^{2}}{\Delta \theta^{2}}\right] \exp \left[-\frac{\nu^{2}}{\Delta \omega^{2}}\right] \\
& +\sum_{\mathbf{g}} A_{g} \delta\left[\left(\nu+\omega_{r}-\omega_{g}\right)\left(1-v_{0} \cos \theta_{0}\right)\right. \\
& \left.-\omega_{r} v_{0} \eta \cos \varphi\right] .
\end{aligned}
$$

$N_{r}$ is the number of counts in the $r$ th channel of detector normalized by one electron. $\mathrm{A}_{g}$ and $\mathrm{B}$ are the amplitudes of the coherent and incoherent components of the radiation, respectively. According to Eqs. (12)-(17), they are expressed as

$$
\begin{gathered}
A_{g}=\frac{e^{2}}{2 \pi} v_{0} \omega_{r} L_{z}\left|\chi_{g}\left(\omega_{r}\right)\right|^{2}\left|1+\delta_{g}^{-1}\right|^{2}, \\
B=\frac{4 e^{2}}{3 \pi} Z^{2}\left(\frac{e^{2}}{m}\right)^{2} \frac{L_{z}}{\Omega} \ln \frac{137}{Z^{1 / 3}} .
\end{gathered}
$$

Performing the integration over $\nu$ in Eq. (27), we arrive at

$$
\begin{aligned}
N_{r}= & B \Delta \theta^{2} \frac{\Delta \omega}{\omega_{r}}+\frac{1}{\pi^{3 / 2}} \int_{0}^{\infty} \eta d \eta \\
& \times \int_{0}^{2 \pi} d \varphi \exp \left[-\frac{\eta^{2}}{\Delta \theta^{2}}\right] \\
& \times \sum_{\mathbf{g}} A_{g} \exp \left[-\frac{\left(\omega_{g}-\omega_{r}+u_{r} \eta \cos \varphi\right)^{2}}{\Delta \omega^{2}}\right],
\end{aligned}
$$

where

$$
u_{r}=\frac{v_{0} \omega_{r}}{1-v_{0} \cos \theta_{0}} .
$$

In general, the integrals in Eq. (30) need to be calculated numerically. However, within the limits of the approximations used in this work, a simple analytical formula can be derived for the radiation spectrum by using the Jensen inequality

$$
\left\langle e^{A}\right\rangle \geqslant e^{\langle A\rangle}
$$

which is well-known from different applications in statistical physics [26] and is valid for averaging over normalized functions of a statistical distribution. As the result, the following approximate expression can be found

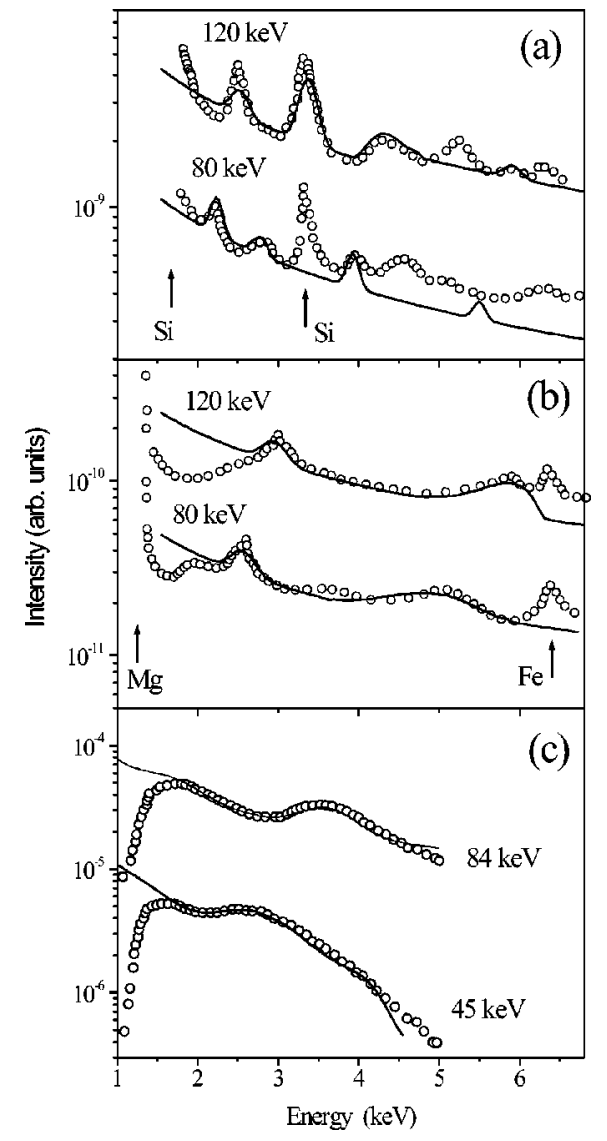

FIG. 4. The experimental data (open dots) reproduced from Ref. [12] (a, Si and b, MgO) and Ref. [11] (c, LiF) and theoretical simulations (solid lines) based on the present theory. The arrows show the emission lines of $\mathrm{Si}, \mathrm{Mg}$ and Fe. For every crystal, two pairs of curves are depicted for different energies of electrons; these illustrate the influence of beam energy on peak position.

$$
\begin{aligned}
N_{r} \simeq & \frac{e^{2}}{2 \pi} L_{z} \Delta \theta^{2}\left|\chi_{0}\left(\omega_{r}\right)\right|^{2} \omega_{r}\left\{\frac{1}{6 \pi^{2}} \omega_{r}^{3} \Omega\left(\ln \frac{137}{Z^{1 / 3}}\right) \frac{\Delta \omega}{\omega_{r}}\right. \\
& +v_{0} \sum_{\mathbf{g}}\left|\frac{\chi_{g}\left(\omega_{r}\right)}{\chi_{0}\left(\omega_{r}\right)}\right|^{2}\left|1+\delta_{g}^{-1}\right|^{2} \\
& \left.\times \exp \left[-\frac{\left(\omega_{g}-\omega_{r}\right)^{2}}{\Delta \omega^{2}}-\frac{\Delta \theta^{2} u_{r}^{2}}{4 \Delta \omega^{2}}\right]\right\}
\end{aligned}
$$

that permits one to investigate the dependence of real CXR spectra on the principal parameters of the experiment. The panels a,b and c of Fig. 4 demonstrate the experimental data (open dots) reproduced from Refs. [11,12] and theoretical spectra (solid lines) simulated by Eq.(31) for crystals of $\mathrm{Si}$, $\operatorname{MgO}\left(\mathbf{v}_{0} \|\langle 111\rangle\right)$ and $\operatorname{LiF}\left(\mathbf{v}_{0} \perp(110)\right)$, respectively. The parameters of the crystals and detectors are shown in the Table

TABLE I. Crystal data and parameters of experiments $[9,10]$.

\begin{tabular}{lccccccc}
\hline \hline Crystal & $a_{0}, \AA$ & $\Delta \omega, \mathrm{keV}$ & $\theta_{0}$ & $\Delta \theta^{2}$, sterad & $L_{z}, \AA$ & $\left|\chi_{0}\right|_{\omega=5 k e V}$ & Orientation \\
\hline Si [12] & 5.4309 & 0.1 & $96^{\circ}$ & 0.05 & 1000 & $4.03 \times 10^{-5}$ & $\mathbf{v}_{0} \|\langle 111\rangle,\langle 100\rangle$ \\
MgO [12] & 4.21 & 0.1 & $96^{\circ}$ & 0.05 & 1000 & $6.03 \times 10^{-5}$ & $\mathbf{v}_{0} \|\langle 111\rangle,\langle 100\rangle$ \\
LiF [11] & 4.0276 & 0.2 & $67.5^{\circ}$ & 0.002 & 1000 & $4.13 \times 10^{-5}$ & $\mathbf{v}_{0} \perp(110),(100)$ \\
\hline \hline
\end{tabular}


I. For every crystal we depict two pairs of curves corresponding to different electron energies, demonstrating the dependence of the peak positions on the velocity of the electrons. The characteristic emission lines of $\mathrm{Si}, \mathrm{Mg}$ and $\mathrm{Fe}$ are indicated by vertical arrows. Slight disagreement of simulations and measurements in the low frequency region is caused by the low-energy threshold of detector sensitivity, which has not been taken into consideration here. Except for this discrepancy, theory and experiment are seen to be in good agreement. This permits us to interpret the experimental results reported in Refs. [11,12] as a confirmation of the contributions of both radiation types: coherent Bremsstrahlung and parametric $\mathrm{x}$-ray radiation, to the coherent output from nonrelativistic electrons. Additionally, the results above indicate the possibility of qualitative and quantitative description of $\mathrm{x}$-ray radiation from nonrelativistic electrons in thin crystals on the basis of perturbation theory. The use of the classical electrodynamics approach allows one to avoid cumbersome calculations based on the multiwave theory of electron diffraction, as treated in Ref. [12].

Although the presented curves do not fit experimental data with accuracy adopted for modern diffraction experiments, the obtained agreement can be considered as acceptable. The probable reasons of discrepancy between theory and experiment are (i) the uncertainty of angular resolution of detectors in experiments $[11,12]$, (ii) the approximate modeling of the instrumental function in Eq. (25), and (iii) the neglect of background radiation from back- and multiplescattered electrons.

Experiments with a high spectral resolution are necessary for more detailed studies on coherent radiation [23]. Then, in accordance with Eq. (27), the exact separation of CXR peaks from other types of radiation could be realized. As one possibility, two- or three-crystal arrangements utilizing additional analyzer crystals might be used.

\section{COMPARISON OF CXR WITH SYNCHROTRON RADIATION}

It follows from the analysis presented, that CXR may be effective for applications where its principal advantages are: i)high spectral intensity of soft $\mathrm{x}$-ray radiation $(1-5 \mathrm{keV})$ in a narrow region near the resonant frequencies and ii)possibility of fine tuning of resonant frequencies. Such features of CXR are important in experiments which require selective influence of $\mathrm{x}$-rays on investigated systems within narrow bandpass and a small integral dose of radiation. Of course, the problems to which CXR may be applied [8], may be successfully tackled by using monochromatized synchrotron radiation (SR). However, the former method has an incontestable advantage of possible realization in the "home" laboratory. The availability of quasi-monochromatic x-ray radiation with tunable frequency from nonrelativistic particles is confirmed, for example, by the results of tabletop $\mathrm{x}$-ray holography, presented recently in the paper by Bompadre [29] et al. The approach proposed in our work can also extend the abilities of PXR from relativistic particles [30] and channeling radiation [31] methods, which are intensively developed by numerous experimental groups.

The key point of replacement of SR by CXR in any specific case is how intense is the output of both methods for
$\mathrm{X}$-rays production. In this section, we present an estimate of the spectral intensities of CXR and SR to evaluate the effectiveness of CXR experiments in comparison with the analogous experiments at a synchrotron facility. The analytical estimates for the ratio of the spectral intensities of CXR and SR normalized for one electron can be derived in the same way as was done in [27]. This expression contains the dependence of spectral intensity on the fundamental parameters of both radiation processes. The full energy loss by one electron during the single cycle within a synchrotron is defined by formula [28]

$$
P_{\omega} \simeq \frac{e^{3} H}{m}
$$

where $H$ is the amplitude of the magnetic field and $\hbar=c$ $=1$. The characteristic frequency and the spectral interval of SR are of the same order

$$
\Delta \omega_{p} \simeq \omega_{p}=\frac{e H}{m}\left(\frac{E}{m}\right)^{2}, \quad E \gg m .
$$

The angular distribution of radiation in the orbital plane is equal $\Delta \theta_{\|} \simeq 1 / 2 \pi$, and in the normal direction $\Delta \theta_{\perp} \simeq m / E$. Then the number of photons emitted in the unit solid angle and registered by a detector with energy resolution $\Delta \omega / \omega$ is equal to

$$
\frac{\partial N_{\omega}^{S R}}{\partial \mathbf{n}}=\frac{1}{\omega} \frac{\partial P_{\omega}}{\partial \mathbf{n}} \simeq \frac{e^{2}}{2 \pi} \frac{m}{E} \frac{\Delta \omega}{\omega} .
$$

The estimate for the analogous value in the case of CXR, i.e., for the number of photons emitted by one electron passing through the crystal is

$$
\frac{\partial N_{\omega}^{C X R}}{\partial \mathbf{n}} \simeq \frac{e^{2}}{2 \pi} \omega L_{z} v_{0}\left|\chi_{g}\right|^{2} .
$$

The smallest possible value of $\Delta \omega / \omega$ is defined by the width of the CXR peak $\left(\omega L_{z}\right)^{-1}$. Taking into account this fact, the ratio of CXR and SR spectral densities can be found as:

$$
\beta=\frac{\partial N_{\omega}^{C X R}}{\partial \mathbf{n}} / \frac{\partial N_{\omega}^{S R}}{\partial \mathbf{n}} \simeq v_{0}\left(\omega L_{z}\right)^{2} \frac{E}{m}\left|\chi_{g}\right|^{2} .
$$

For example, for radiation from the spectral series (220) of an LiF crystal and an electron of energy $63 \mathrm{keV}$, the ratio is $\beta \simeq 10^{-2}$. The resonant frequency of a synchrotron for that case corresponds to an electron energy of $2 \mathrm{GeV}$ with a magnetic field of magnitude 1.2 Tesla [24].

The spectral density of PXR from relativistic electrons, being compared to the spectral density of SR, gives [27] the value $\beta \gg 1$. However, the average current in a synchrotron is of order $1 \mathrm{~A}$, this is $10^{7}-10^{9}$ times higher than the current in a conventional linear accelerator, where PXR is usually observed [2,4-6]. The absolute intensity of SR from an electron beam is hence considerably higher than the intensity of PXR. Meanwhile, the current of power x-ray tubes is reaching values comparable with the average current of synchrotrons. Parameter $\beta$ thus permits us to compare the abilities of laboratory equipment with a synchrotron facility. It should be emphasized, that the spectral densities of synchrotron ra- 
diation and parametric x-ray radiation (or, generally speaking, coherent $\mathrm{x}$-ray radiation) are comparable within a narrow spectral interval. With regard to the integral power of a $\mathrm{SR}$, it is much higher than the power of CXR, in according with estimation derived in Ref. [8].

\section{CONCLUSIONS}

On the basis of classical electrodynamics we have investigated the theory of coherent $\mathrm{x}$-ray radiation from nonrelativistic electrons in crystal. Two components contribute to the intensity of CXR peaks, parametric X-ray radiation and coherent Bremsstrahlung. This study essentially completes the results, reported recently in work by Morokhovskyi [16] et al., where analogous phenomenon have been observed for relativistic particles. The analysis of CXR spectra from lowenergy particles requires us to account for the following additional peculiarities: isotropic angular distribution of radiation, influence of background and instrumental detector function.

On the basis of the derived formulas, we interpreted the experiments on radiation from nonrelativistic electrons in crystal $[11,12]$, which have not been quantitatively explained previously. Good agreement between theoretical and experimental curves confirms the validity and completeness of the theory used for the description of electromagnetic processes within the crystal. The necessity of background and detector function consideration should be pointed out as well. The results presented can also be treated as experimental confirmation for the application of CXR from nonrelativistic electrons as a source of quasi-monochromatic $\mathrm{x}$-ray radiation with tunable frequency and as a new method for structure analysis [8]. In particular, the structure factors depending on atomic coordinates contribute to total CXR intensity in a different way than structure factors which influence the reflection intensities of conventional x-ray diffraction measurements. Thus, the simulataneous analysis of x-ray scattering from the sample and $\mathrm{x}$-ray radiation from nonrelativistic electrons passing through the sample opens a new approach to the solution of the phase problem in crystallography, supplementing other direct methods of phase determination [32].

Finally, the following possible applications of CXR can be designated: (i) the possibility to easily vary the CXR frequency extends the abilities of the anomalous dispersion method for phase determination within elementary cells, containing no heavy atoms [33], (ii) a comparison of the spectral densities of CXR and synchrotron radiation indicates possible advantages of the former for the investigation of systems within a narrow spectral interval, where the integral dose of radiation plays a crucial role, (iii) the analysis of CXR spectra from nonrelativistic electrons striking the sample at grazing angle is of special interest for defect investigations.

\section{ACKNOWLEDGMENTS}

The authors thank Dr. J. D. Ferrara of the Molecular Structure Corp. and Dr. V. Kaganer (Paul-Drude-Institute, Berlin) for comments on the manuscript. One of us (I.D.F.) acknowledges the Belarussian Foundation for Fundamental Researches (Grant Nos. Ф97/244, Ф97/099). J.C.H.S. acknowledges NSF Grant No. DMR9814055.
[1] M.L. Ter-Mikaelian, High Energy Electromagnetic Processes in Condensed Media (Wiley, New York, 1972).

[2] V.G. Baryshevskii and I.D. Feranchuk, Zh. Eksp. Teor. Fiz. 61, 944 (1972) [Sov. Phys. JETP 34, 502 (1972)].

[3] G.M. Garybyan and C. Yang, Zh. Éksp. Teor. Fiz. 61, 430 (1972) [Sov. Phys. JETP 34, 495 (1972)].

[4] Y.N. Adishchev, V.G. Baryshevskii, S.A. Vorobiev, V.A. Danilov, S.D. Pak, A.P. Potylizyn, P.F. Safronov, and I.D. Feranchuk, Pis'ma Zh. Éksp. Teor. Fiz. 41, 295 (1985) [Sov. Phys. JETP 41, 361 (1985)].

[5] K.-H. Brenzinger, B. Limburg, H. Backe, S. Dambach, H. Euteneuer, F. Hagenbuck, C. Herberg, K.H. Kaiser, O. Kettig, G. Kube, W. Lauth, H. Schöpe, and Th. Walcher, Phys. Rev. Lett. 79, 2462 (1997).

[6] S. Asano, I. Endo, M. Harada, S. Ishii, T. Kobayashi, T. Nagata, M. Muto, K. Yoshida, and H. Nitta, Phys. Rev. Lett. 21, 3247 (1993).

[7] J. Freudenberger, V.B. Gavrikov, M. Galemann, H. Genz, L. Groening, V.I. Morohovskii, V.V. Morohovskii, U. Nething, A. Richter, J. Sellschop, and N.F. Shulga, Phys. Rev. Lett. 74, 2487 (1995).

[8] I.D. Feranchuk and A. Ulyanenkov, Acta Crystallogr., Sect. A: Found. Crystallogr. A55, 466 (1999).

[9] O.R. Frisch and D.N. Olson, Phys. Rev. Lett. 3, 141 (1959).

[10] A.W. Saenz and H. Uberall in Coherent Radiation Sources, edited by A.W. Saenz and H. Uberall (Berlin, Springer-Verlag, 1985)

[11] Y.S. Korobochko, V.F. Kosmach, and V.I. Mineev, Zh. Éksp. Teor. Fiz. 48, 1248 (1965) [Sov. Phys. JETP 21, 834 (1965)].

[12] G.M. Reese, J.C.H. Spence, and N. Yamamoto, Philos. Mag. A 49, 697 (1984)

[13] I.D. Feranchuk and A.V. Ivashin, J. Phys. (France) 46, 1981 (1985).

[14] S.V. Blazhevich, G.L. Bochek, V.N. Gavrikov, V.I. Kulibaba, N.I. Maslov, N.N. Nasonov, B.H. Pirogov, A.G. Safronov, and A.V. Torgovkin, Phys. Lett. A 195, 210 (1994).

[15] V.L. Kleiner, N.N. Nasonov, and A.G. Safronov, Phys. Status Solidi B 181, 223 (1994).

[16] V.V. Morokhovskyi, J. Freudenberger, H. Genz, V.L. Morokhovskii, A. Richter, and J.P.F. Sellschop, Phys. Rev. B 61, 3347 (2000).

[17] V.G. Baryshevskii and I.D. Feranchuk, J. Phys. (France) 44, 913 (1983)

[18] V.G. Baryshevskii, Channelling, Radiation and Reactions in Crystals for High Energies (Belarussian University, Minsk, 1982).

[19] V.G. Baryshevskii and I.D. Feranchuk, Dokl. Akad. Nauk BSSR 18, 499 (1974)

[20] J.C.H. Spence and G. Reese, Acta Crystallogr., Sect. A: Found. Crystallogr. A42, 577 (1986).

[21] H. Nitta, Phys. Lett. A 158, 270 (1991). 
[22] C.J. Humphreys and J.C.H. Spence, in Proceedings EMSA, edited by G.W. Bailey, (Claitor's, Louisiana, 1979), 554.

[23] J.C.H. Spence and M. Lund, Phys. Rev. B 44, 7054 (1991).

[24] M. Hart, 23rd Course on X-ray and Neutron Dynamical Diffraction (NATO Advanced Study Institute, Erice, 1996), pp. $85-103$

[25] A.I. Akhiezer and V.B. Berestetzkii, Quantum Electrodynamics (Nauka, Moscow, 1969).

[26] R.P. Feynman, Statistical Mechanics (W.A. Benjamin, Massachusetts, 1972)

[27] V.G. Baryshevskii and I.D. Feranchuk, Nucl. Instrum. Methods Phys. Res. B 228, 490 (1985).

[28] V.L. Ginzburg, Theoretical Physics and Astrophysics (Nauka, Moscow, 1975).
[29] S.G. Bompadre, T.W. Petersen, and L.B. Sorensen, Phys. Rev. Lett. 83, 2741 (1999).

[30] R.B. Fiorito, D.W. Rule, X.K. Maruyama, K.L. DiNova, S.J. Evertson, M.J. Osborne, D. Snyder, H. Rietdyk, M.A. Piestrup, and A.H. Ho, Phys. Rev. Lett. 71, 704 (1993).

[31] B.L. Berman, J. Kephart, R. Pantell, S. Datz, H. Park, R. Klein, and B. Dahling in Relativistic Channeling, Vol. B165 of NATO Advanced Studies Institute Series, edited by R. Carrigan and J. Ellison (NATO Advanced Studies Institute, Erice, 239, 1987).

[32] Q. Shen, Phys. Rev. B 59, 11109 (1999).

[33] I.D. Feranchuk and A.V. Ivashin, Kristallografiya 34, 39 (1989). 